\title{
CONTACT:
}

- Thomas Houston, MD, MPH

Thomas.houston@umassmed.edu

Phone: 508-856-8999

- Thomas English, PhD

Thomas.english@umassmed.edu

Phone: 508-856-8958 


\section{Clinical Research Informatics}

Thomas Houston, MD, MPH May 4, 2012 


\section{Informatics at UMMS/UMMHC}

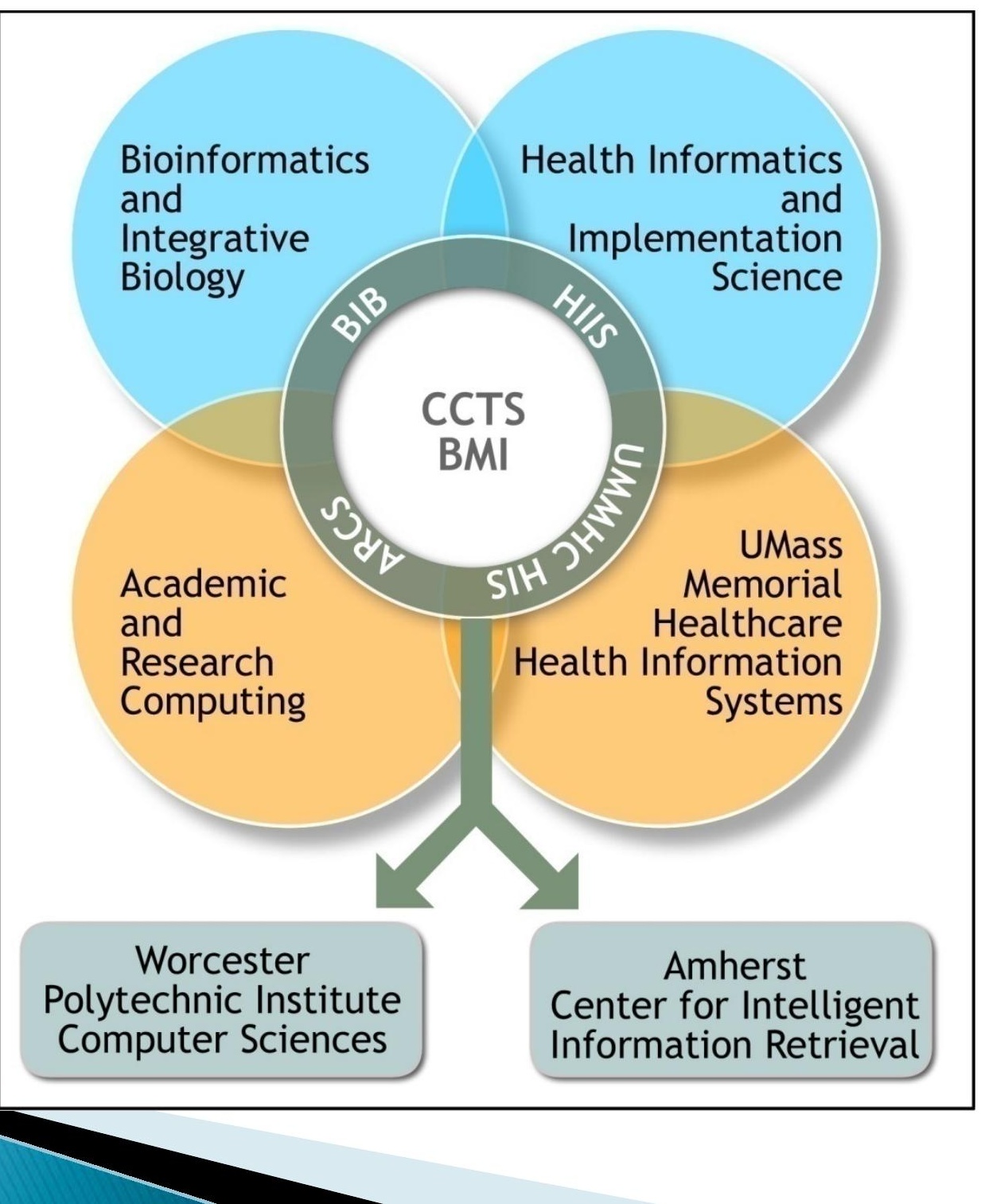




\section{BRIDG}

Biomedical Research Informatics Development Group

- Tom Houston, MD, MPH, Chief, Division of Health Informatics and Implementation Science, Quantitative Health Sciences

- Rajani Sadasivam, PhD

- Thomas English, PhD

- Ralph Zottola, PhD

- Timothy Hogan, PhD 


\section{Informatics}

- Discipline focused on the acquisition, storage, and optimal use of information for health and healthcare

- "Informatics is more about information than technology, with the latter being a tool, albeit an important one, to make best use of information."

- Hersh BMC Medical Informatics 2009 


\section{Health IT}

- HIT = the application of computers and technology in health care settings

- Hersh BMC Medical Informatics 2009 


\section{Clinical research Informatics}

- "Development, application, and evaluation of theories, methods, and systems to optimize the design and conduct of clinical research and the analysis, interpretation, and dissemination of the information generated."

- Embi et al Clinical research informatics: challenges, opportunities and definition for an emerging domain. J Am Med Inform Assoc 2009 

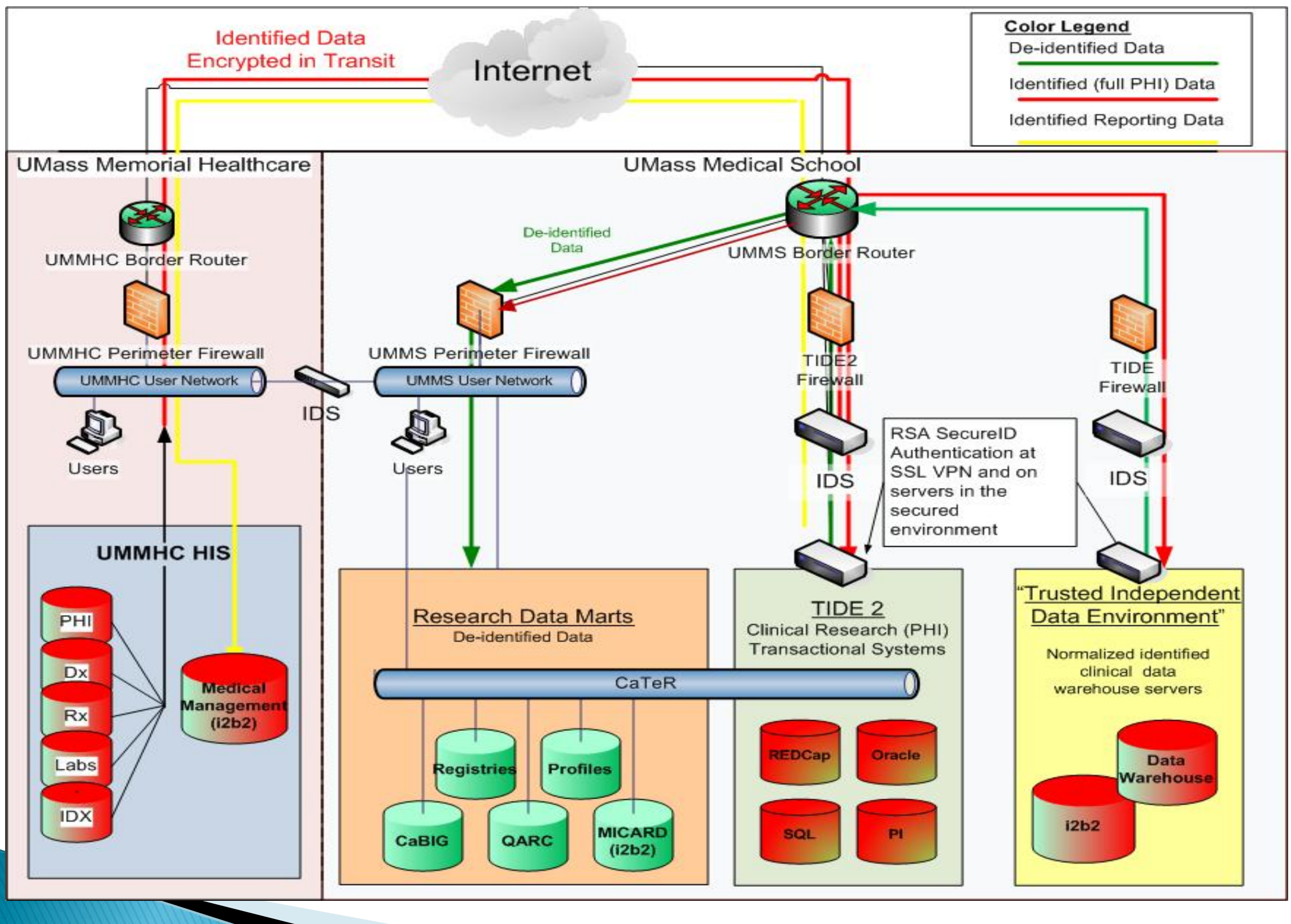


\section{Development}

- Usability Assessments

- Expert-driven evaluation

- User-driven evaluation

- Quantitative (time on task)

- Qualitative (cognitive think-aloud)

- Jakob Nielson. Designing Web Usability: The Practice of Simplicity, 2000. 


\section{Personal Health Records (PHRs)}

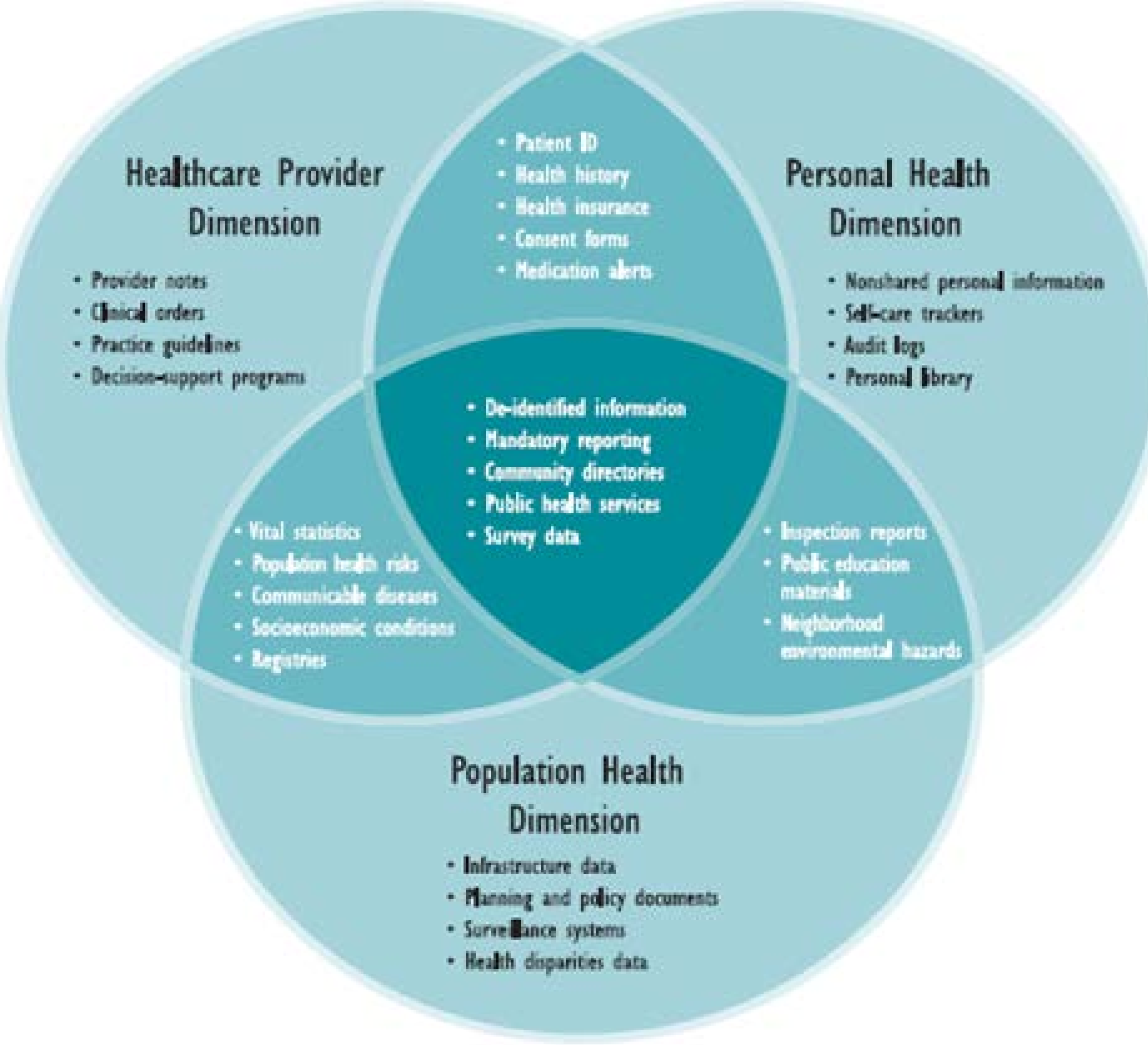

"A set of computerbased tools that allow people to access and coordinate their lifelong health information and make appropriate parts of it available to those who need it." 


\section{The Potential of PHRs:}

The Chronic Care Model

- Enhanced Patient Satisfaction

- Patient Activation

- Enabled Self-Management

- Enhanced Communication

- Efficiency and Cost

- Quality and Safety

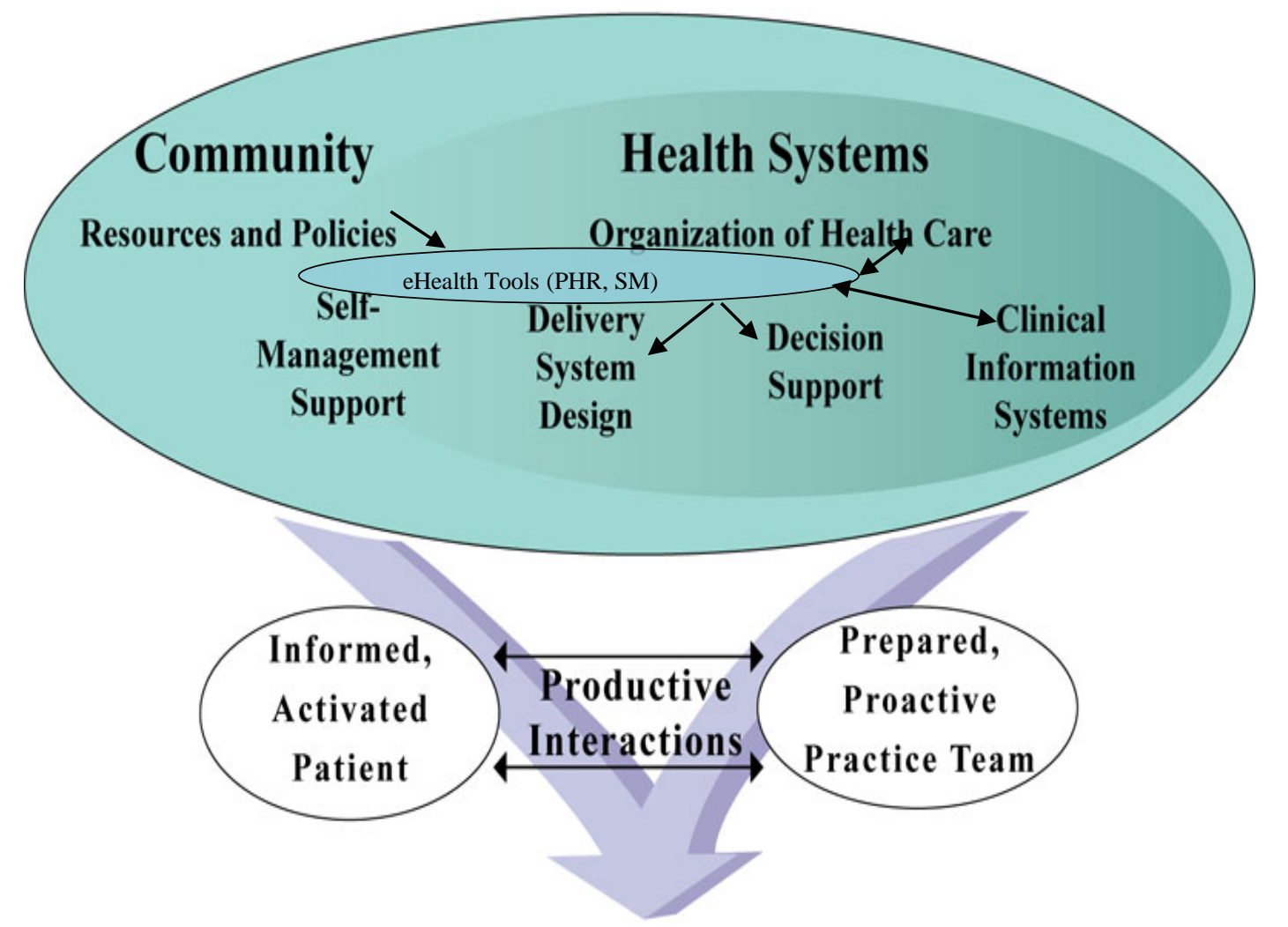

Improved Outcomes 


\section{Disparities}

Cumulative Proportion by Month of KPGA Enrollees Registered with KP.org from 10/1/05.

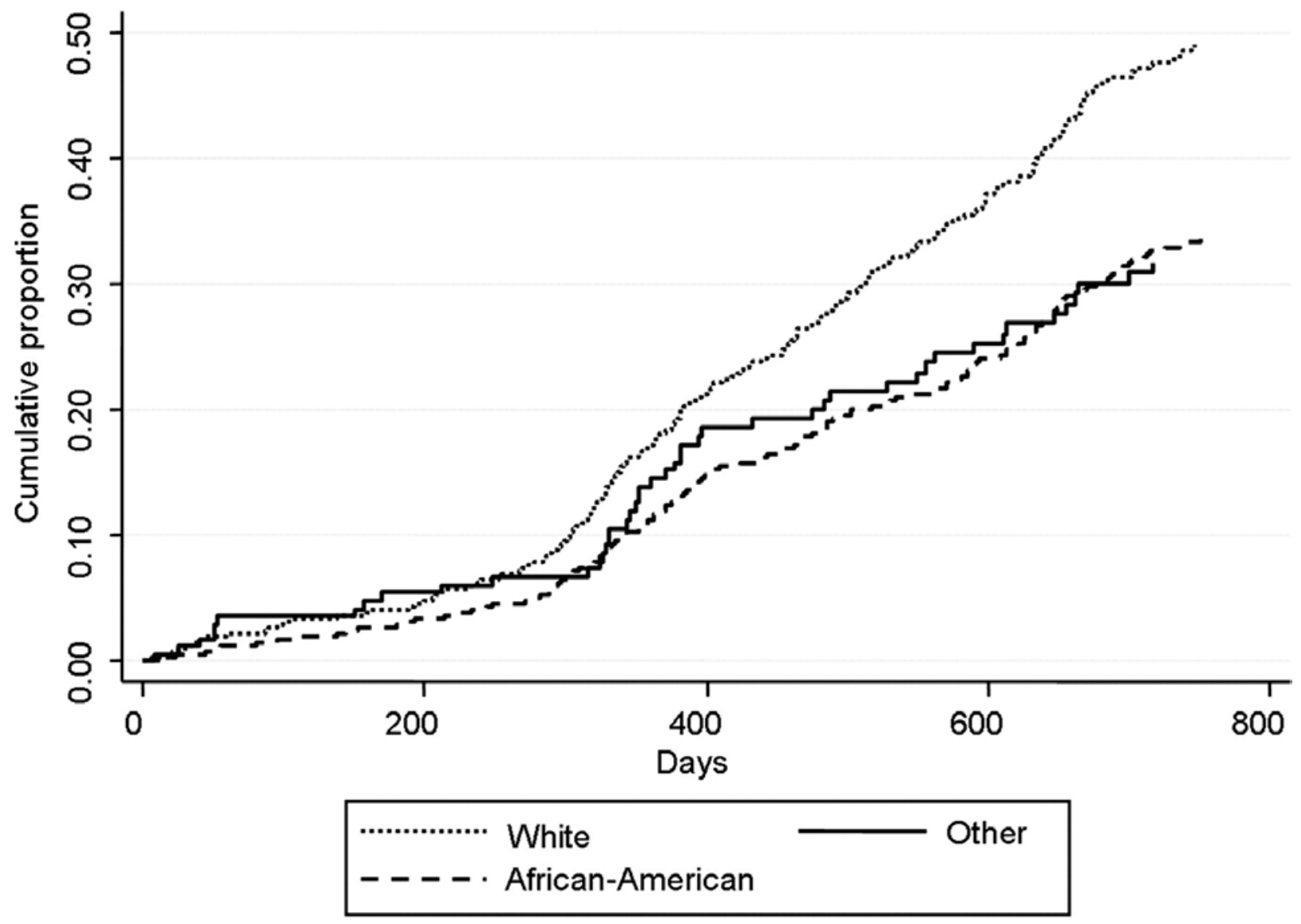

Roblin, Houston et al. JAMIA 2009;16:683-689 
Ecosystem of applications making use of a patient's or a population's PCHR data

\section{PCHR platform}

Subscription agents for standards-based exchange

Data providers

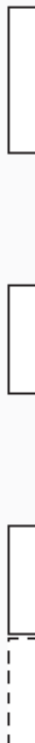

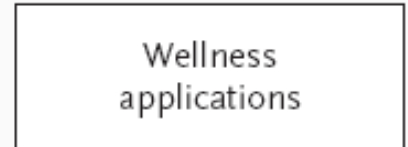

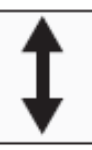

Clinical researchmanagement tools

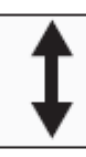

Interface to applications $\uparrow$

Information access controls (personally controlled)

Secure storage of lifelong persistent data across all sources

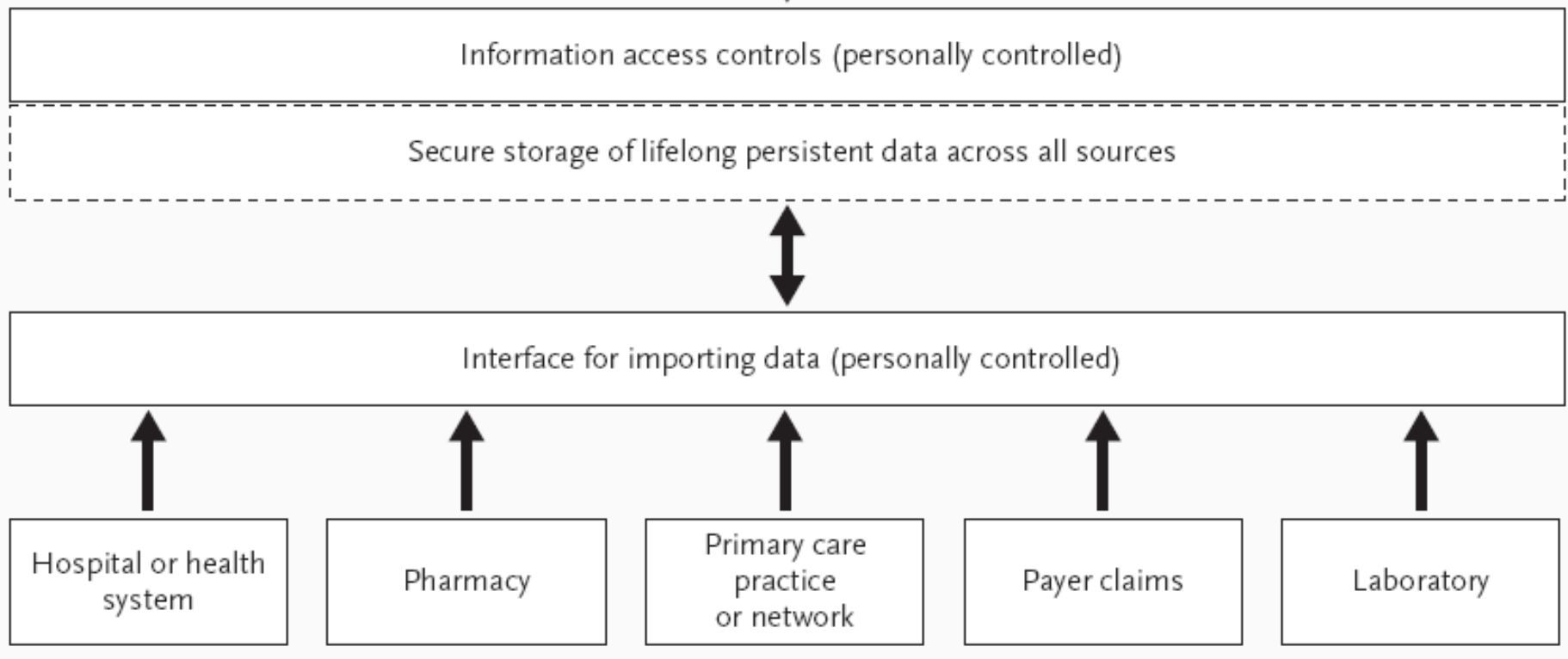

Interface for importing data (personally controlled)

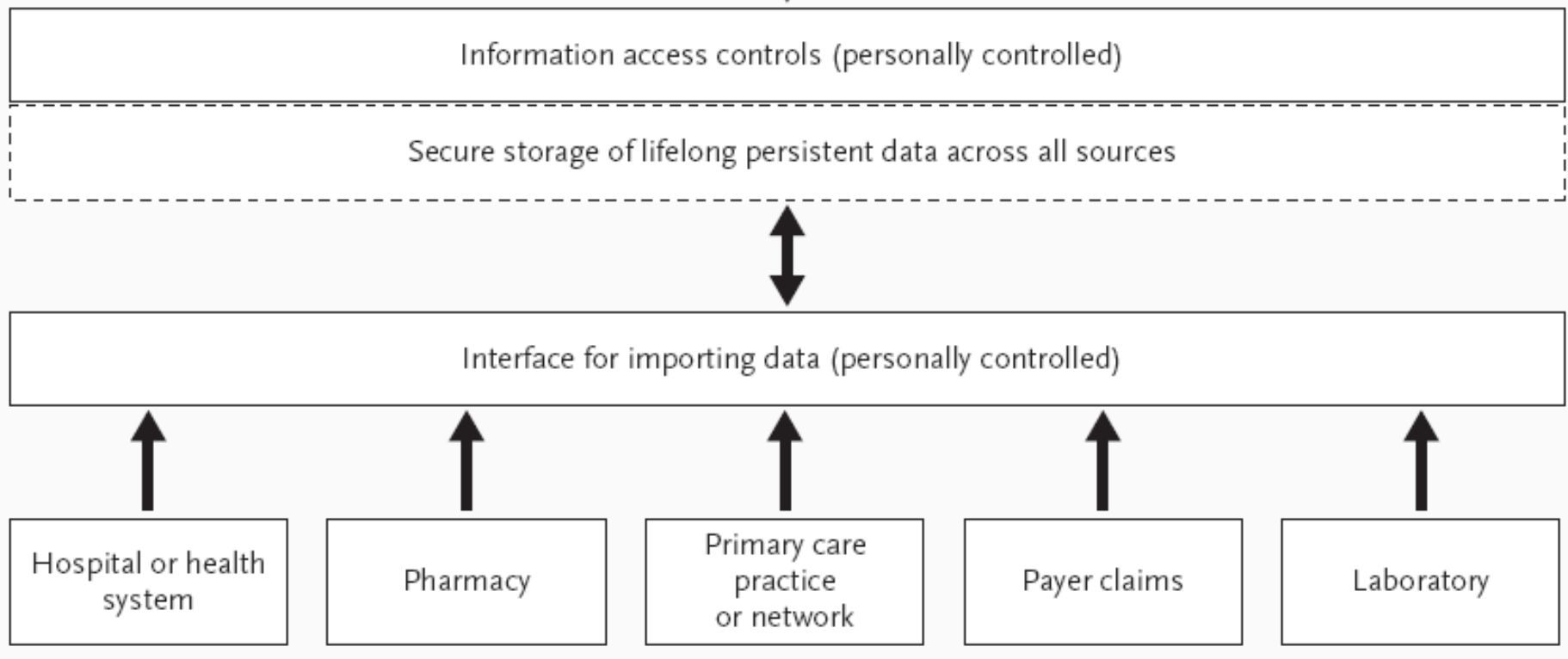

Social-networking tools

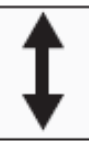

Mandl et al, NEJM 2008 
Collect patient information, such as self-reported demographic and risk factor information (health behaviors, symptoms, diagnoses, and medications)

2 Integrate patient information with clinical information through links to the electronic medical record and/or claims data

Interpret clinical information for the patient by translating

3 clinical findings into lay language and delivering health information via a user-friendly interface

Provide individualized clinical recommendations to the patient,

4 such as screening reminders, based on the patient's risk profile and on evidence-based guidelines

Facilitate informed patient action integrated with primary and specialty care through the provision of vetted health

5 information resources, decision aids, risk calculators, personalized motivational messages, and logistical support for appointments and follow-up
Krist and Woolf, JAMA, 2011 


\section{eHealth QUERI}

- Leadership

- Tom Houston, MD, MPH (Director)

- Bonnie Wakefield, PhD, RN, FAAN (Clinical Coordinator)

- Timothy Hogan, PhD (Implementation Research Coordinator)

- Field Sites

- Boston, Chicago, Salt Lake, Ann Arbor, Portland, lowa City

Data Center (West Haven VAMC) 


\section{eHealth QUERI Strategic Goals}

1) To augment access and meaningful use of eHealth tools

2) To enhance veteran self-management and participation in collaborative care through the design, evaluation, and implementation of appropriate eHealth tools 


\section{Using Allscripts Data at UMass for Clinical Research}

Thomas English PhD

Thomas.english@umassmed.edu

Department of Quantitative Health Sciences Division of Health Informatics and Implementation Science

University of Massachusetts Medical School

Center for Clinical and Translational Science

Quantitative Methods Core: Bruce Barton PhD

Biomedical Informatics Core: Tom Houston MD

$4 / 20 / 2011$ 


\section{Purpose}

- Discuss the role Allscripts can play in your research

- Discuss how to access Allscripts data 


\section{Who am I?}

- Health Informatics \& Outcomes Researcher

- Former Allscripts Administrator

- Former Allscripts Analytics Administrator

- Design Studies

- Modify Systems

- Extract Data

- Analyze Data 


\section{How can Allscripts Help?}

- Grant Preparation

- Cohort Discovery

- Data source

- Intervention 


\section{(}

国 (All Types) - Filter by Type $\mathrm{E}_{\mathrm{Q}}$ View English Query Help.

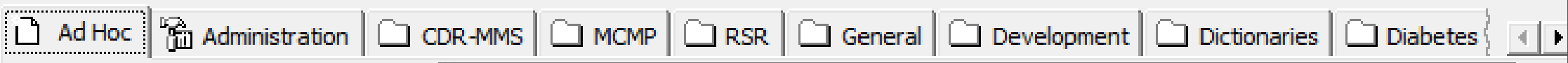

Analytics Worksheets

\#\$ Analytics Analysis

3if Analytics Charts

畾 Analytics Custom Reports

蜆 Analytics Access Center

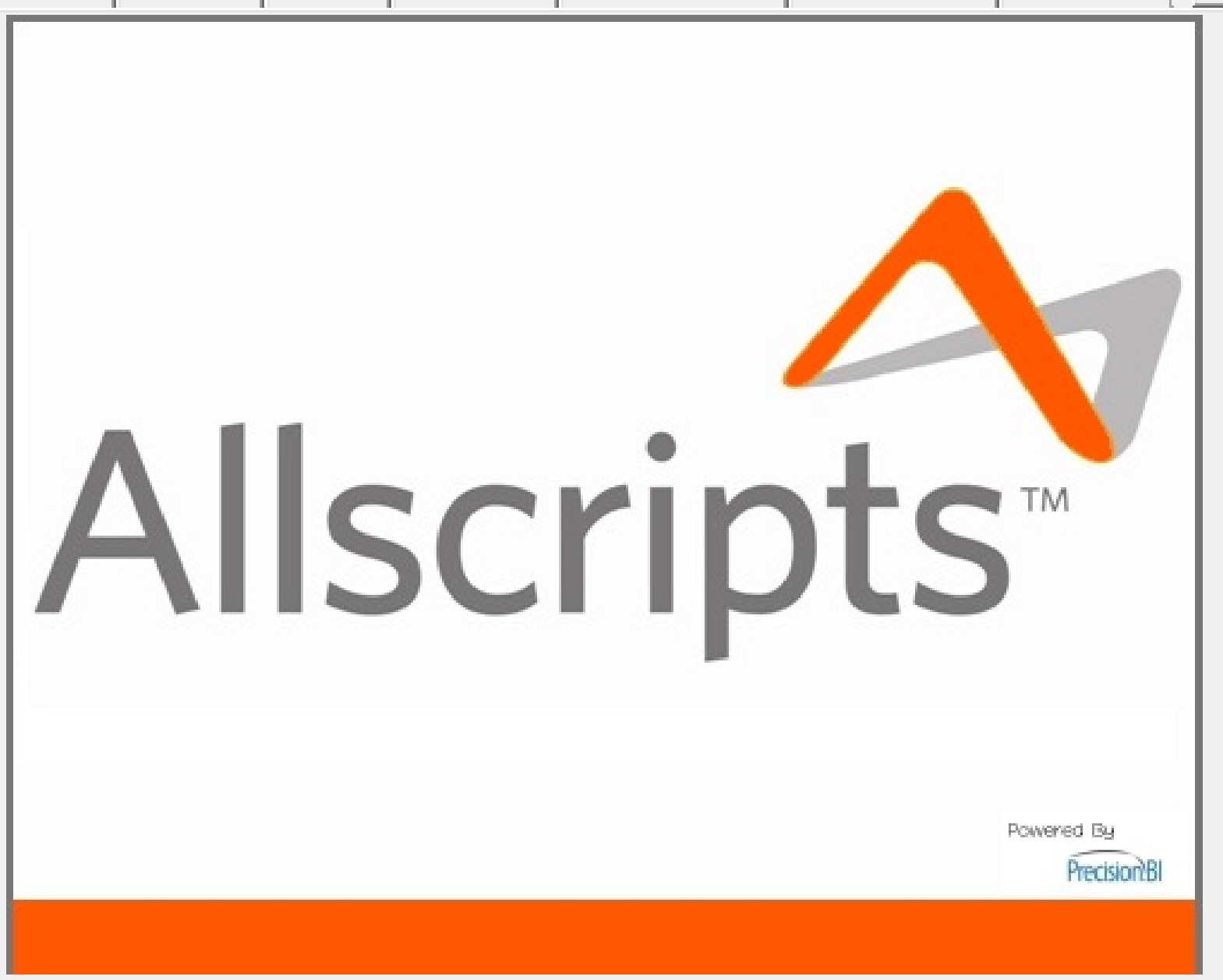


Grant Preparation 


\section{Grant Preparation}

- Some data can be gathered prior to IRB approval

- Power Calculations

- Sample Size

- Summary Statistics

- All research using Allscripts will be subject to IRB approval

- Individual data will not be gathered without prior IRB approval 


\section{Population Characteristics}

- Race/Ethnicity

- Age

- Medications

- Lab results

- Immunizations

- Comorbidities

- Data about individuals will not be available until the IRB has approved the study. 
Cohort Discovery 


\section{New England Newborn \\ Screening Program}

- How many children have:

- SCID, DiGeorge Syndrome, Velo-Cardio-Facial Syndrome etc...

- Pulled cohorts from Micard and Allscripts

- Challenges:

- ICD9 Codes

- Matching Terminology 


\section{Focused Recruitment}

- Suppose we did need to recruit the patients for the study. How would we?

- With Allscripts we can extract patient demographics including contact info. Mail, call, email...

Or

- Query the system to find out when potential participants will be at the clinic and approach them individually. 
Data Source Example 


\section{Metformin \& B12}

- Resident research with no funding

- Can you pull the number of subjects we follow with diabetes who:

1. Are treated with metformin

2. Had a vitamin B12 level drawn within 2 years prior to starting metformin. 


\section{Metformin \& B12}

- Explore the effect of Metformin on Vitamin B12 levels

- Initial Plan: Find patients on Metformin that had a B12 level checked within 24 months prior to the start of Metformin.

- Contact the patients and repeat the Serum B12 if they are willing

- We found 1700 patients meeting the criteria 


\section{Metformin \& B12}

- 1700 Potential Participants

- But I did some digging.

- I found 600 of those patients had a serum B12 level after the start of Metformin already documented.

- We now have IRB approval to extract the data from Allscripts to conduct the analysis

- Now we can do the study using data already in the system as part of standard care. No recruiting! No Specimen Management! 


\section{B12 (6386 Results) Issues}

- What do you do with non numeric values?

- $<50$

- $>1500$

- Average B12

- 665.9

-What do the different codes mean?
- hB12
$\mathrm{n}=3963$
Average: 643
- $\mathrm{h} * * \mathrm{~B} 12 \quad \mathrm{n}=2415$
Average: 704 


\section{ED Visits}




\section{Emergency Department Visits}

- Investigate trends and predictors of ED use

- Claims data

- Allscripts data

- Problems

- Medications

- Hospitalizations

- \# Visits

- Lab Results

- Etc.....

- Data about physician and clinic characteristics

- Create predictive model to determine who is most likely to use the ED 


\section{ED Visit and No Other Care.}

- $\mathrm{N}=25844$

- Male:52\% Female: 48\%

Age Group

$(<1)$

(1-10)

(11-19)

(20-39)

$(40-64)$

$(65+)$ n

457

5584

3584

6668

5965

3586
Percent

$1.77 \%$

$21.61 \%$

$13.87 \%$

$25.80 \%$

$23.08 \%$

$13.88 \%$ 


\section{ED Visit Race Data}

Race

American Indian or Alaskan Native

Asian

Asian or pacific

Black or African American

Hispanic-

Multi Racial

Native Hawaiian or Other Pacific Islander

Other Race

White

Unknown n

12

296

5

929

25

2

16

1805

12749

10017
Percent

$0.05 \%$

$1.15 \%$

$0.02 \%$

$3.59 \%$

$0.10 \%$

$0.01 \%$

$0.06 \%$ $6.98 \%$ $49.33 \%$ $38.76 \%$ 


\section{ED Visit Count?}

25844 Patients had 39265 ED Visits

Numbers represent the number of "Emergency Department " document items in Allscripts.

May have duplicates and/or additional ED reports on the same visit.

\# of Documents

1
2
3
4
$>=5$

\% Population

$73.19 \%$

$16.51 \%$

$5.36 \%$

$2.24 \%$

$2.70 \%$
$\%$ Visits

$48.17 \%$

$21.73 \%$

$10.59 \%$

$5.91 \%$

$13.60 \%$

$>5$ shows 697 patients had 5340 ED reports 


\section{Allscripts as Part of an Intervention}




\section{AAFP Foundation Childhood}

Immunizations Project

- Goal: Improve Childhood Immunization Rates

- Intervention involved patient education, physician education, and tools enable by Allscripts.

- Used IDX, the scheduling system, to remind nurses to review immunizations on patients 19-35 months old.

- Used Allscripts to create lists of patients not up to date for physician to review and for us to contact in bulk using mailers

- Also gave quality scores to all physicians and benchmarked them against their peers 


\section{Childhood Immunizations}

- Immunization rate increases significantly

- Largely due to finding missing documentation of Hep B vaccine given at the hospital

- 2010 AAFP Foundation Pfizer Immunization Best Practice Award 


\section{Closing the Feedback Loop}

- AHRQ Grant

- Made Follow-up calls to patients presenting with acute illness

- Compared Human Calls vs. Automated Calls

- Analysis is under way

- Extracted data from Allscripts to gather participant contact info

- Added specific task types in Allscripts to inform physicians about their patients we called and how the patient was doing 


\section{Allscripts Diabetes}

- Natural Experiment around Allscripts Implementation

- Investigated Diabetes Care Pre/Post Allscripts Implementation

- Analyzed data using Orders, Results, and Lab Values

- Allows us to find patients that have tests ordered but do not have them performed. 


\section{Allscripts Diabetes}

- Post EHR Implementation patients were more likely to have an:

- $\mathrm{HbA} 1 \mathrm{c}<7$

- $\mathrm{LDL}<100$

- A Microalbumin Ordered

- Data Collection 1 month vs. 4 hours 


\section{Intervention Ideas}




\section{CKD Report}

\section{Card}

generated in

Allscripts

Analytics will be part of an intervention.
Renal Medicine

\section{Patient Name: TEST, MARY $\quad$ PCP:} Patient MRN: 1506618

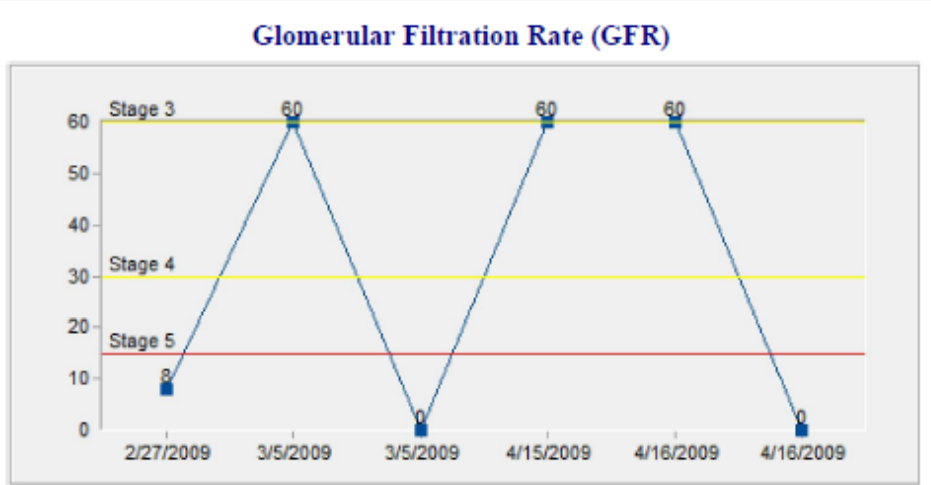

Stage 1 Kidney Damage Normal Function GFR $>90$

Stage 2 Kidney Damage Mild Funtion Decrease GFR 60-90

Stage 3 Kidney Damage Modrate Function Decrease GFR 60-30 Stage 4 Kidney Damage Severe Function

Decrease GFR 15-30 Stage 5 Kidney Failure GFR $<15$

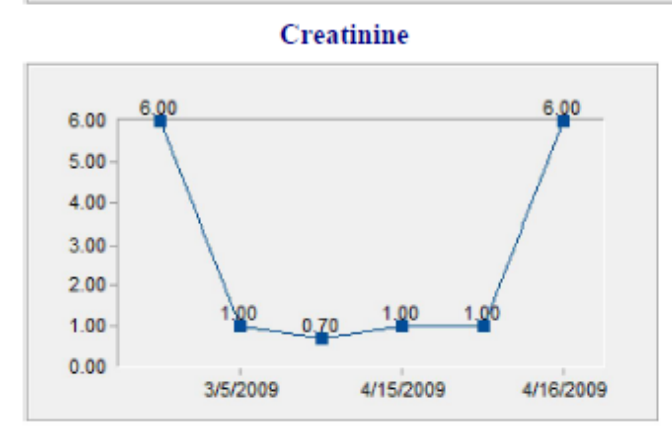

Other Labs Results Date Goal

oal

\begin{tabular}{|llll|}
\hline Protein Urine & & & \\
MicroAlbumin & 1 & $3 / 5 / 2009$ & \\
Calcium & 9.0 & $4 / 16 / 2009$ & \\
Sodium Bicarbonate & & & \\
Phosphorus & 2.6 & & \\
PTH & & & $>30$ \\
Vitamin D & 40 & $3 / 5 / 2009$ & $3.5-5.4$ \\
Potassium & 5.0 & $4 / 17 / 2009$ & \\
Serum Albumin & 3.7 & & $>10$ \\
Hemoglobin & 14.0 & $11: 34: 00$ & $<7 \%$ \\
Alc & 4.0 & $3 / 5 / 2009$ & \\
\hline
\end{tabular}

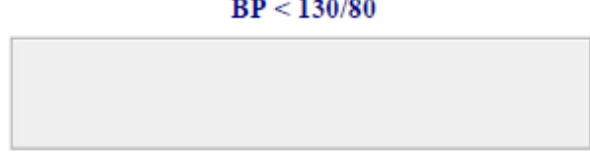

Diet Instructions

Limit dietary sodium to $2 \mathrm{~g} / 24 \mathrm{hrs}$. Avoid salty snacks, canned soups, processed and fast food

Limit dietary potassium to $2 \mathrm{~g} / 24 \mathrm{hrs}$. Limit tomatoes and tomato sauce, oranges and orange juice, bananas, potatoes (boiled potato is $\mathrm{OK}$ ), avocadoes and cantaloupes.

\section{Medications}

ACE Inhibitor Accupril 5 MG Oral Tablet
ARB
Lipid Lowering

Avoid Use of NSAID and other Nephrotoxic Medications

\section{Recommended Referrals}




\section{Pragmatic Trial}

- Grant Stipulates data must be a residual of usual care.

- Use Allscripts and patient portal to improve care

- Extract Allscripts data

- Tasks

- Documents

- Orders

- Patient reported outcomes

- Allscripts based tools

- Customize Allscripts workflows 
Summary 


\section{Limitations}

- Missing Data

- Noisy Data

- Linking Concepts to Data

- Confounding - by indication, by severity, etc Variations in data entry by provider and clinical setting

- Different Allscripts Functionality in UMass clinics 


\section{Strengths}

- Real-world data

- Huge volumes of data

- 622,254 Patients with an arrived visit since 1/1/2007

- 2,396,711 Blood Pressures on 318418 patients

- Inexpensive to retrieve (relative to direct capture) Dually valuable for research and operations

- Access to grant opportunities calling for electronic data that is the residual of usual care 
So, how does this work? 


\section{Services Available}

- It is best to integrate Allscripts into your research from the beginning!!!

- Allscripts data and capabilities will affect:

- Collect pilot data

- Analysis of existing data

- Study design

- Intervention Design

- Grant preparation (sample size/power, analysis plans, QA/QC, data management, DSMB)

- Data management

- Data Coordinating Center (DCC) activities

- Analytic Approach

- In the near future we should be able to do similar work with Soarian 


\section{Cost}

- Partially supported by the CTSA

- Junior investigators and short consults are generally at no cost (CTSA)

- Help with grant applications is generally at no cost

- Adequate support must be in the grant budget

- Long-term analyses projects require support

- There are various mechanisms, including P01 program/project grants

- Let's talk 


\section{Contact Info}

- Tom English PhD

- Thomas.english@umassmed.edu

- 508.856.8958

- Bruce Barton PhD

- Quantitative Methods Core

- Bruce.Barton@UMassMed.edu

- QMC@UMassMed.edu 